\section{CASE REPORT}

doi: 10.5455/medarh.2018.72.295-296

MED ARCH. 2018 AUG; 72(4): 295-296

RECEIVED: JUL 04, 2018 | ACCEPTED: AUG 02, 2018

Saudi Board of Otorhinolaryngology, Department of Otorhinolaryngology, Head and Neck Surgery, King Fahad General Hospital -ALMusaedeah Center, Jeddah-Saudi Arabia

Corresponding author: Mohammed AI Sheikh. Department of Otorhinolaryngology, Head and Neck Surgery, King Fahad General Hospital -ALMusaedeah Center, Jeddah-Saudi Arabia. ORCID ID: http://www.orcid.org: : 0000-00019941-5377. E-mail: sheikh@gmail.com
(C) 2018 Mohammed Al Sheikh, Saad Alenzi, Asma Al Ahmadi, Ali Mashragi, Hoda Alsayid, Abdullah Alamri

This is an Open Access article distributed under the terms of the Creative Commons Attribution NonCommercial License (http://creativecommons.org/ licenses/by-nc/4.0/) which permits unrestricted non-commercial use, distribution, and reproduction in any medium, provided the original work is properly cited.

\title{
Actinomycosis of the Middle Ear and Mastoid
}

\author{
Mohammed Al Sheikh, Saad Alenzi, Asma Al Ahmadi, Ali Mashragi, Hoda \\ Alsayid, Abdullah Alamri
}

\section{ABSTRACT}

Introduction: Actinomycosis is a saprophytic infection caused by actinomycetes. Actinomycetes is a gram positive, anaerobic, non acid-fast, filamentous bacterium. Although actinomyce is considered as a part of the normal flora, it is rarely seen in middle ear and mastoid cavity. Aim: is to report a rare case of Actinomycosis infection of the middle ear and mastoid cavity. Case report: We presented A 24 years old male arrived to our outpatient department complaining of right-sided otalgia and hearing loss for 8 years, no history of otorreah, vertigo or dizziness. He had a history of right-sided chronic suppurative otitis media with a history of two operations at the same side. This report suggests that actinomycosis, although it is rare, it could occurs in middle ear. It should be considered as one of the differential for chronic suppurative otitis media patients with no improvement on medical treatment. Conclusion: Combined medical and surgical treatment is the recommended management for Actinomycosis infection of the middle ear and mastoid cavity.

Key words: Actinomyces, Mastoid, Actinomycosis, Ear, Middle.

\section{INTRODUCTION}

Actinomycosis is a saprophytic infection caused by actinomycetes. Actinomycetes is a gram positive, anaerobic, non acid-fast, filamentous bacterium. Usually considered as apart of the normal flora. In human, actinomycosisis raeli is the most common pathogenic species of this bacteria. There are many important factors to be considered in pathogenesis of actinomycosis infections, such as trauma due to dental extraction, manipulation or caries. These factors provide an entry for these organisms to go deep into tissue where anaerobic conditions are necessary for their growth. It is commonly seen after minor trauma in cervicofacial region (55\%) as well as the abdomen and thorax. Involvement of the middle ear and mastoid is rare and might be caused by direct spread via the eustachian tube from the nasopharynx.

\section{CASE REPORT}

A 24 years old male arrived to our outpatient department complaining of right-sided otalgia and hearing loss for 8 years, no history of otorreah, vertigo or dizziness. He had a history of right-sided chronic suppurative otitis media with a history of two operations at the same side, which were right side atticotomy combined with tympanoplasty in 2002 and right side tympanoplasty in 2007. Examination showed dull, intact tympanic membrane. Tuning fork showed mild conductive hearing loss. The rest of the examination was within normal.

Audio-tympanogram showed mild conductive hearing loss in the right side and type B tympanogram. A computed tomography (CT) scan showed opacification of the right middle ear and mastoid as shown in Figure 1. Patient was admitted to the hospital and all pre-operative hematological, biochemical and serological investigations were within normal. Patient underwent exploratory right tympanatomy combined with right cortical mastoidectomy. Tissue specimens were sent for histopathology and comprehensive microbiology for aerobic, anaerobic, fungal and acid-fast bacillus cultures.

Histopathology showed acute and chronic inflammation with diffuse fibrosis. Acid-fast bacillus staining was negative and all microbiological cultures failed to grow any organisms. A Grocott-Gomori's methenamine silver-nitrate stain (GMS stain) showed branching bacterial filaments as seen in Figure 2, allowing the diagnosis of actinomycosis to be established.

Patient had a history of Penicillin allergy. Consultations for both infectious diseases and allergy/immunology specialties were obtained to determine an optimal management strategy. He was started on Augmentin 1g and Doxycy- 


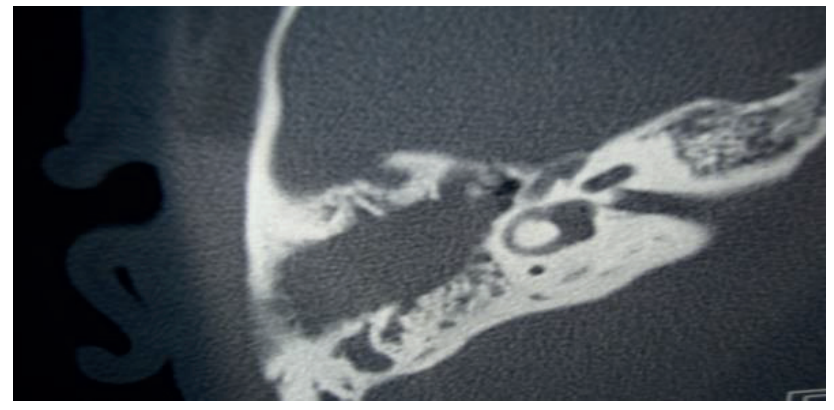

Figure 1. Pre-operative CT scan showing middle ear and mastoid opacification

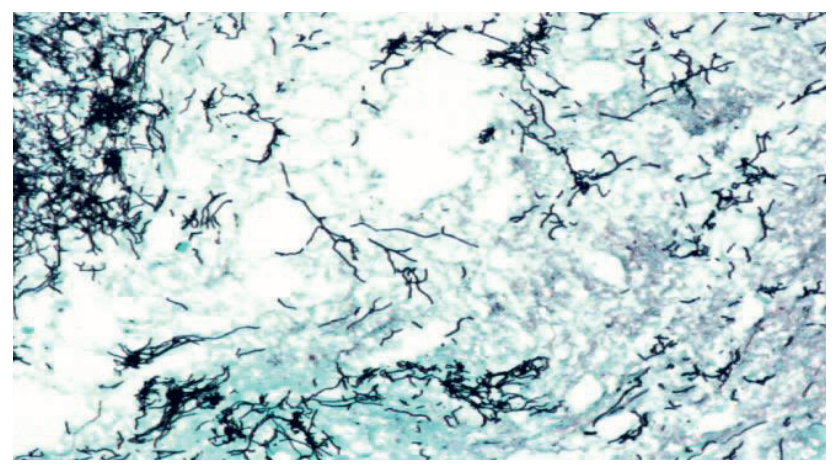

Figure 2. Grocott-Gomori's methenamine-silver nitrate stain showing cluster of branching bacterial filaments of actinomycosis

cline $200 \mathrm{mg} /$ day. Then, discharged in a stable condition on same medications with regular outpatient clinic appointments. During regular follow-up visits in outpatient clinic till up to date patient has no complaint, tympanic membrane looked normal. CT was done showed normal right middle ear and mastoid opacification as shown in Figure 3.

\section{DISCUSSION}

Actinomycosisis a subacute or chronic disease due to infection by actinomycesIsraeli, which is usually characterized by abscess formation, tissue fibrosis and draining sinuses. This commensal organism is found normally in high concentration in tonsils and carious teeth. It is considered as an opportunistic pathogen. In 1878, actinomycosis in human was first described by Israeli and Wlfeand. First, they isolated the causative agent in a culture. Then they defined the organism's anaerobic nature. Before antibiotic era, diagnosis was often made at autopsy, as most of these infections were fatal because of their intracranial complications. Currently these cases usually presented as a chronic suppurative otitis media with prolonged course of treatment. In literature, most of these cases needed an intact canal wall tympanomastoidectomy followed by prolonged antibiotic treatment.

Isolating actinomyces species from cultures of surgical specimen makes most accurate diagnosis. However, $70 \%$ of these cultures are negative because it is fastidious organism. Granules of actinomycosis strongly support the diagnosis, which are usually seen in histological examination. It is rarely visible on hematoxylin-eos in stained sections but can be seen as round or oval basophilic masses with a radiating arrangement of eosinophilic terminal "clubs", Or using of other staining such as Grocott-gomori's methenamine-silver nitrate stain, maccallen-good posture stain or brown-brenn stain and p-aminosalicylic acid. Combination of medical and surgical treatment is recommend-

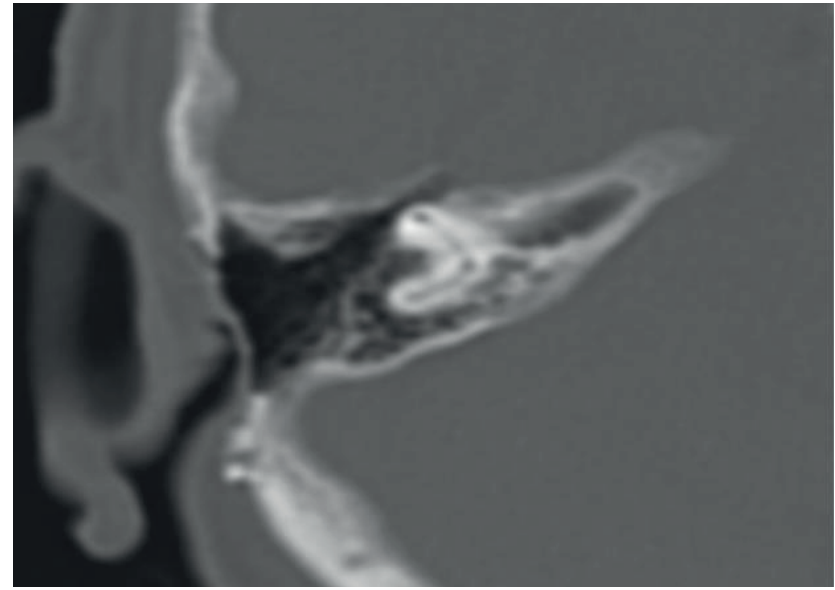

Figure 3. Post-operative CT scan showing normal opacification of middle ear and mastoid

ed. Surgery is performed first for mechanical debridement of the affected area to reduce bacterial load. Followed by prolonged course of medical therapy. Recommended treatment regimes vary from six weeks to one year depending on the site and severity of the infection. Actinomycesspecies are usually susceptible to a number of antimicrobials therapies. Including Penicillin, Chloramphenicol, Tetracyclines, Erythromycin, Clindamycin, Streptomycin, Imipenem and Cephalosporins. Prolonged observation is necessary to detect recurrences.

\section{CONCLUSION}

Although actinomyce is considered as a part of the normal flora, it is rarely seen in middle ear and mastoid cavity. This study suggests that actinomycosis, although it is rare, it could occurs in middle ear. It should be considered as one of the differential for chronic suppurative otitis media patients with no improvement on medical treatment. Combined medical and surgical treatment is the recommended management.

- Declaration of patient consent: Authors certify that they have obtained patient consent form.

- Conflict of interest: none declared.

- Author's contribution: M.A. main surgeon and reviewing the writing of the case report and first author, S.H.A take the history of the patient and examination, A.M. did all investigation needed before OR, A.A.A. following the patient up and wring the case report, H.A.A. reviewing the writing of the case report and all information of the patient, A.A. reviewing the writing of the case report and all information of the patient.

\section{REFERENCES}

1. Olson TS, SeidAB, Pransky SM. Actinomycosis of the middle Ear. International Journal of Pediatric Otorhinolaryngology. 1989; 17: 51-55.

2. Shelton C, Brackmann DE. Actinomycosis Otitis Media. Arch Otolarnyngology Head Neck Surg. 1988; 111: 88-89.

3. Leek JH. Actinomycosis of the Tympanomastoid. Laryngoscope. 1974; 184: 290-301.

4. Shaheen SO, Ellis FG. Actinomycosis of the larynx. J R Soc Med. 1983; 76: 226.

5. Bartels LJ, Vrabec DP. Cervicofacial Actinomycosis: a Variable Disorder. Arch Otolaryngol. 1978; 110: 705.

6. Samuels RHA, Martin MV. A Clinical and Microbiological Study of Actinomycetes in Oral and Cervicofacial lesions. Br J Oral Maxillofac Surg. 1988; 126: 458.

7. Harris LF, Kakani PR, Selah CE. Actinomycosis: Surgical Aspects. Am J Surg. 1985; 51: 262. 